\title{
HEMOGLOBINOPATIAS EM GESTANTES SUBMETIDAS AO TESTE DA MÃEZINHA NA REDE PÚBLICA DE SAÚDE
}

\author{
HEMOGLOBINOPATHIES IN PREGNANT WOMEN SUBMITTED TO THE MOMMY TEST IN THE \\ BRAZILIAN PUBLIC HEALTH NETWORK
}

\author{
Keesi Marcela Matos Liberato ${ }^{\mathrm{a}^{*}}$, Gleidson Brandão Oselame ${ }^{\mathrm{b}^{* *}}$, Eduardo Borba Neves $^{\mathrm{c}^{* *}}$ \\ akee.si@hotmail.com, bgoselame@ics.curitiba.pr.gov.br, cborbaneves@hotmail.com \\ *Centro Universitário Campos de Andrade - Curitiba (PR), Brasil \\ **Universidade Tecnológica Federal do Paraná - Curitiba (PR), Brasil
}

Data de recebimento do artigo: 16/09/2016

Data de aceite do artigo: 14/10/2016

\section{RESUMO}

Introdução: As hemoglobinopatias estão entre as doenças genéticas de grande relevância no mundo. Aproximadamente $7 \%$ da população mundial apresenta algum tipo de hemoglobinopatia, e estimativas apontam que a cada ano nascem cerca de 300 a 400 mil crianças portadoras desses distúrbios. Objetivo: Descrever os diagnósticos prevalentes de hemoglobinopatias em gestantes submetidas ao Teste da Mãezinha na rede pública de saúde. Métodos: Estudo descritivo transversal com análise quantitativa dos dados. Foram avaliados 1.013 prontuários eletrônicos de gestantes vinculadas à rede de saúde pública do município de Pinhais, estado do Paraná, no período entre 25 de fevereiro de 2014 e 25 de fevereiro de 2015. Foram incluídos prontuários de gestantes do primeiro ao terceiro trimestre de gestação que realizaram o Teste da Mãezinha. Resultados: Verificou-se que 55,7\% das gestantes realizaram o Teste da Mãezinha no $1^{\circ}$ trimestre de gestaçáo. Apenas $0,7 \%$ dos resultados do teste apresentaram traço de Hemoglobina C e $1,3 \%$ traço falciforme. Quanto a raça, nos resultados dos exames alterados $(\mathrm{n}=20), 50 \%$ eram brancos, $40 \%$ pardos e $10 \%$ negros. Dentre estas gestantes, $80 \%$ apresentaram intercorrências na gestação, sendo mais frequente a infecção do trato urinário (50\%), leucorreias em geral (25\%) e intercorrências durante o parto (12,5\%). Conclusáo: Neste estudo observou-se uma baixa prevalência de hemoglobinopatias. Recomenda-se a implantaçáo do Teste da Mãezinha na rede de saúde pública brasileira para o diagnóstico precoce das hemoglobinopatias gestacionais.

Palavras-chave: Doença da hemoglobina SC; traço falciforme; hemoglobina falciforme; anemia falciforme.

\section{ABSTRACT}

Introduction: The hemoglobinopathies are among genetic diseases of great importance in the world. Approximately 7\% of the global population have some kind of hemoglobinopathy, and estimates show that about 300,000 to 400,000 children affected by these disorders are born each year. Objective: To describe prevalent diagnoses of hemoglobinopathies in pregnant women submitted to the Mommy Test in the Brazilian public health network. Methods: Cross-sectional, descriptive study with quantitative analysis of the data. The sample included 1,013 electronic medical records of pregnant users of the public health network of the municipality of Pinhais, state of Paraná, Brazil, recorded between February $25^{\text {th }}, 2014$ and February $25^{\text {th }}, 2015$. Medical records of pregnant women undergoing up until the third quarter of pregnancy submitted to the Mommy Test were used. Results: It was observed that $55.7 \%$ of the pregnant women were submitted to the Mommy Test in their first quarter. Only $0.7 \%$ of the results of the test showed the Hemoglobin C trait, and $1.3 \%$ presented sickle cell trait. As for race, in the results that showed alterations $(n=20), 50 \%$ of the women were white, $40 \%$ were brown, and $10 \%$ were black. Among these participants, $80 \%$ presented intercurrences during the pregnancy, the most frequent being urinary tract infection (50\%), general leucorrhea (25\%), and intercurrences during delivery (12.5\%). Conclusion: A low prevalence of hemoglobinopathies was observed in this study. The implementation of the Mommy test in the Brazilian public health network is recommended for early diagnosis of gestational hemoglobinopathies.

Keywords: Hemoglobin SC disease; sickle cell trait; sickle cell hemoglobin; sickle cell anemia. 


\section{Introdução}

As hemoglobinopatias estão entre as doenças genéticas de grande relevância no mundo ${ }^{1}$. Entre elas destaca-se a doença falciforme (DF) que acomete predominantemente os afrodescendentes. Aproximadamente $7 \%$ da população mundial apresenta algum tipo de hemoglobinopatia, e estimativas apontam que nascem por ano de 300 a 400 mil crianças acometidas por esses distúrbios, das quais 250 mil são $\mathrm{DF}^{2}$.

Nesse sentido, a população brasileira, por ser caracterizada por uma mistura acentuada e diversificada de raças, apresenta elevada prevalência de hemoglobinopatias $^{3,4}$. No Brasil, estima-se que existam mais de sete milhões de pessoas portadoras do traço falciforme, e 25 a 30 mil com a doença falciforme (DF). No Paraná, de 2002 a 2004 a prevalência de doença falciforme foi de 2,2:100.000 nascidos vivos, com maior número de casos na 2a Regional de Saúde (Metropolitana) e na 15a Regional (Maringá) 2,5 .

A doença ou o traço falciforme, a hemoglobina C e as talassemias alfa e beta são as hemoglobinopatias predominantes no Brasil. Diante disso, a Organização Mundial da Saúde sugere programas de prevenção e controle dessas doenças em toda a América Latina ${ }^{4,5}$.

Um exemplo de doença falciforme é a anemia falciforme, doença hereditária que causa uma mutação no ponto da globina beta da hemoglobina, originando no lugar da hemoglobina $\mathrm{A}(\mathrm{HbA})$ uma hemoglobina mutante, denominada hemoglobina $\mathrm{S}(\mathrm{HbS})^{6}$. Essa doença é caracterizada por episódios repetidos de vaso-oclusão, que são causados pelas hemácias falciformes (com formato de foice) e acarretam diversas complicaçóes clínicas que afetam todos os órgãos e/ou tecidos, crises álgicas, febre e quadro infeccioso ${ }^{2}$.

De acordo com a deliberação no 330 de 24 de novembro de $2012^{7}$, que amplia a oferta de exames de pré-natal, o protocolo da Rede Mãe Paranaense determina a realizaçáo de exame de eletroforese de hemoglobina pelo método focalização isoelétrica para detectar hemoglobinopatias, anemia falciforme e traços de talassemias precocemente. No Paraná, a Lei Estadual 867/1987 , tornou obrigatório o Teste do Pezinho, que consiste em triagem para fenilcetonúria em todos os recém-nascidos do estado. O Ambulatório de Hematologia Pediátrica do Hospital de Clínicas da Universidade Federal do Paraná, em Curitiba, e toda a Rede Hemepar, que conta com 22 unidades espalhadas por todo o estado do Paraná, são serviços conveniados. Recentemente, em dezembro de 2013, iniciou-se no estado do Paraná a pesquisa de hemoglobinopatias em gestantes, o Teste da Mãezinha².
O Programa de Prevenção das Hemoglobinopatias em Gestantes no Paraná (PPHG) é coordenado pela Secretaria de Estado de Saúde (SESA-PR) e tem como objetivo um conjunto de açôes voltadas à atenção e cuidado da gestante e da criança durante o primeiro ano de vida?

De acordo com o PPHG, mais conhecido como Teste da Mãezinha, gestantes de mais de 300 municípios do Paraná têm o direito de realizar gratuitamente o teste para diagnóstico precoce de hemoglobinopatias, como a doença falciforme e a talassemia major, assim como o tratamento dos casos identificados ${ }^{6}$. Nesse sentido, o objetivo deste estudo foi descrever os diagnósticos prevalentes de hemoglobinopatia em gestantes submetidas ao Teste da Máezinha na rede pública de saúde.

\section{Método}

Trata-se de um estudo do tipo descritivo, retrospectivo, documental, com análise quantitativa de dados. Segundo Gil ${ }^{10}$, a pesquisa exploratória tem como objetivo adequar os problemas e torná-los mais específicos, e o uso descritivo do estudo tem como característica a padronizaçáo da coleta de dados de uma populaçáo por meio de questionário ou prontuário. $\mathrm{O}$ estudo retrospectivo consiste na aquisição de dados de um período de tempo para discussão e análise ${ }^{11}$. A avaliação quantitativa é caracterizada pela coleta de informaçóes, interpretação e análises estatísticas dos dados.

Os dados foram coletados na Prefeitura Municipal de Pinhais, por meio da base de dados e prontuário eletrônico do Departamento de Assistência à Saúde. Foram incluídos dados de 1.013 gestantes, em qualquer fase gestacional (do primeiro ao terceiro trimestre de gestação) que realizaram o Teste da Mãezinha. Foram excluídas gestantes não vinculadas ao programa de pré-natal do município. Os dados utilizados referem-se aos exames realizados no período entre 25 de fevereiro de 2014 e 25 de fevereiro de 2015.

Foram seguidos os preceitos éticos em pesquisa envolvendo seres humanos conforme determina a Resolução no 466/2012 ${ }^{12}$ do Conselho Nacional de Saúde. A pesquisa foi aprovada pelo Comitê de Ética em Pesquisa do Centro Universitário Campos de Andrade, sob parecer consubstanciado no 1.446 .391 de 2016, e autorizada pela Secretaria de Saúde do município de Pinhais.

Os dados colhidos foram armazenados em um banco de dados para posterior análise estatística por meio do software Statistical Package for the Social Sciences (SPSS). Utilizou-se a estatística descritiva na análise das variáveis. 


\section{Resultados}

Perfil das gestantes que realizaram o Teste da Mãezinha

Das 1.013 gestantes cujo prontuário foi analisado, $563(55,7 \%)$ realizaram o Teste da Mãezinha no $1^{\circ}$ trimestre de gestaçăo. Apenas 20 gestantes apresentaram alteraçóes como traço hemoglobina $\mathrm{C}$ e traço falciforme. Essas e as demais variáveis são apresentadas na Tabela 1.

Tabela 1: Coletas do teste para hemoglobinopatias, com idade gestacional, resultados alterados e amostras inadequadas $(n=1.013)$, Pinhais, 2016.

\begin{tabular}{|lcc|}
\hline \multicolumn{1}{|c}{ Variáveis } & n & $\%$ \\
\hline Idade gestacional da coleta do exame & & \\
\hline $1^{\circ}$ trimestre (1 a a $13^{\mathrm{a}}$ semanas) & 563 & 55,7 \\
\hline $2^{\circ}$ trimestre (14 a a $27^{\mathrm{a}}$ semanas) & 350 & 34,4 \\
\hline $3^{\circ}$ trimestre (28 a a 40a semanas) & 34 & 3,4 \\
\hline Sem informação da idade gestacional & 66 & 6,5 \\
\hline Resultados alterados e amostras inadequadas & & \\
\hline Normal & 978 & 96,5 \\
\hline Traço Hemoglobina C & 7 & 0,7 \\
\hline Traço falciforme & 13 & 1,3 \\
\hline Amostra inadequada & 14 & 1,4 \\
\hline Não mora no município & 1 & 0,1 \\
\hline
\end{tabular}

\section{Análise de prontuários das portadoras de traço falciforme e traço hemoglobina $C$}

Foram analisados 20 prontuários que apresentaram alteraçóes no teste no período gestacional das portadoras de traços falcêmico ou hemoglobina C. Foram avaliados dados como etnia, idade, histórico gestacional, referência gestacional de baixo ou alto risco, intercorrências no pré-natal e tipo de parto.

Destaca-se que 75\% ( $n=15)$ das gestaçóes foram de baixo risco, sendo realizado o pré-natal na Unidade de Saúde da Família (USF). As intercorrências durante o período de gestação entre as gestantes com resultado positivo para traço falciforme ou hemoglobina $\mathrm{C}$ foram de $80 \%(n=16)$. Ainda, $15 \%(n=3)$ não tiveram seguimento do pré-natal devido a mudança de município. A Tabela 2 apresenta essas variáveis.
Tabela 2: Análise dos prontuários das gestantes com alterações no Teste da Mãezinha (n=20), Pinhais, 2016.

\begin{tabular}{|c|c|c|}
\hline Variáveis & $\mathbf{n}$ & $\%$ \\
\hline \multicolumn{3}{|l|}{ Etnia } \\
\hline Branca & 10 & 50 \\
\hline Parda & 8 & 40 \\
\hline Negra & 2 & 10 \\
\hline \multicolumn{3}{|l|}{ Idade } \\
\hline $15-20$ & 6 & 30 \\
\hline $21-25$ & 6 & 30 \\
\hline $26-30$ & 5 & 25 \\
\hline $31-35$ & 2 & 10 \\
\hline $36-40$ & 1 & 5 \\
\hline \multicolumn{3}{|l|}{ Histórico gestacional } \\
\hline $1^{\text {a }}$ gestação & 10 & 50 \\
\hline 2 a 3 gestações & 8 & 40 \\
\hline 4 a 5 gestaçóes & 2 & 10 \\
\hline \multicolumn{3}{|l|}{ Histórico de abortos } \\
\hline Abortos & 3 & 15 \\
\hline Sem histórico de aborto & 17 & 85 \\
\hline \multicolumn{3}{|l|}{ Referência gestacional } \\
\hline Baixo risco & 15 & 75 \\
\hline Alto risco & 5 & 25 \\
\hline \multicolumn{3}{|l|}{ Gestação atual } \\
\hline Com intercorrências & 16 & 80 \\
\hline Sem intercorrências & 1 & 5 \\
\hline Sem segmento no pré-natal & 3 & 15 \\
\hline \multicolumn{3}{|l|}{ Tipo de parto } \\
\hline Normal & 10 & 50 \\
\hline Cesárea & 5 & 25 \\
\hline Sem anotaçōes & 5 & 25 \\
\hline
\end{tabular}

\section{Resultados de intercorrências}

gestacionais nas portadoras de traço falciforme e hemoglobina $C$

Observou-se alto índice de intercorrências nas gestantes portadoras de traços falcêmico ou hemoglobina C. A infecção do trato urinário teve maior índice nas gestantes analisadas $(24,2 \% ; \mathrm{n}=8)$. As demais intercorrências estão listadas na Tabela 3. 
Tabela 3: Análise dos prontuários das gestantes com intercorrências na gestação (traço falciforme ou hemoglobina C) no Teste da Mãezinha (n=16), Pinhais, 2016.

\begin{tabular}{|lcc|}
\hline \multicolumn{1}{c}{ Intercorrências na gestaçáo } & n & $\%$ \\
\hline Asma & 1 & 6,25 \\
\hline Anemia & 1 & 6,25 \\
\hline Bradicardia fetal & 1 & 6,25 \\
\hline Hematêmese & 1 & 6,25 \\
\hline Hipocromia & 1 & 6,25 \\
\hline Infecção das vias aéreas superiores & 1 & 6,25 \\
\hline Infecção do trato urinário & 8 & 50,00 \\
\hline Intercorrência no parto & 2 & 12,5 \\
\hline Labirintite & 1 & 6,25 \\
\hline Leucorreias em geral & 4 & 25,00 \\
\hline Osteossintese & 1 & 6,25 \\
\hline Perda de peso & 1 & 6,25 \\
\hline Prematuridade & 1 & 6,25 \\
\hline Pielonefrite & 1 & 6,25 \\
\hline Reumatismo & 1 & 6,25 \\
\hline Sem anotações do parto & 5 & 31,25 \\
\hline Sífilis & 1 & 6,25 \\
\hline
\end{tabular}

\section{Discussão}

Dos 1.013 resultados do Teste da Máezinha avaliados, $34,4 \%$ foram realizados no $2^{\circ}$ trimestre e $3,4 \%$ no $3^{\circ}$ trimestre de gestação, o que pode ser considerado um índice desfavorável. Destaca-se que a captação das gestantes com realização da primeira consulta de pré-natal deve ser até 120 dias do início da gestação ${ }^{13}$. O Teste da Mãezinha é indicado para triagem diagnóstica das hemoglobinopatias, tendo sua indicação de realização no primeiro trimestre de gestação nas unidades básicas de saúde (UBS) dos municípios credenciados pela Fundação Ecumênica de Proteção ao Excepcional ${ }^{6}$.

Em um estudo também realizado na rede pública de saúde, que teve como objetivo caracterizar gestantes em atendimento pré-natal e os fatores associados à adesão no primeiro trimestre de gestação, identificou-se que $40 \%$ das gestantes procurou a Unidade Básica de Saúde para vinculação ao programa por ser mais próximo de sua residência, $60 \%$ por ser totalmente gratuito, e $86,7 \%$ declarou satisfaçáo com o atendimento ofertado ${ }^{14}$.

Portanto, é necessária a captaçáo precoce das gestantes para a realização do teste, pois caso o resultado apresente alteraçóes, a gestante será encaminhada para orientaçáo ou acompanhamento de alto risco.

Quanto aos tipos de hemoglobinopatias que foram detectados com o Teste da Mãezinha, 1,3\% apresentou o traço falciforme e traço de hemoglobina C $(0,7 \%)$. Os portadores do traço falciforme (HbAS) ou traço hemoglobina $\mathrm{C}(\mathrm{HbAC})$ possuem apenas um gene defeituoso ${ }^{15}$. Esses genes costumam ser assintomáticos, inclusive com hemograma normal, porém, suas hemácias não são completamente normais, mas em circunstâncias desfavoráveis podem sofrer transformação em hemácias falcêmicas ${ }^{2,5,6}$. Não se identificou no estudo nenhum diagnóstico de doença falciforme ou talassemias no período da análise. Esse índice pode ser considerado baixo, pois no Brasil há mais de 7 milhões de pessoas portadoras do gene da hemoglobina $\mathrm{S}(\mathrm{HbS})$ no estado heterozigótico (chamadas de portadoras do traço falciforme), com prevalência na populaçáo geral de 2 a $8 \%$, e de 25 a 30 mil com doença falciforme ${ }^{2,5}$.

Destaca-se que a doença falciforme acarreta uma gestação de alto risco, caracterizada por crises de infecções, necessidades de transfusóes de sangue e possibilidade de parto prematuro. Já o traço falciforme caracteriza-se quando uma pessoa herda apenas um gene da hemoglobina $S$. As gestantes portadoras do traço falciforme devem seguir as orientaçóes dadas no pré-natal ${ }^{6}$.

Sobre a presença de amostras inadequadas, apenas $1,4 \%$ apresentou erros no processo de coleta do Teste da Mãezinha. Esse índice pode ser considerado baixo, e ocorre geralmente por envelhecimento do sangue ou coleta inadequada. $\mathrm{O}$ teste é realizado em papel filtro com uma gota do sangue da gestante. A ficha de coleta apresenta dois círculos, que devem ser preenchidos com sangue, verificando se estáo completamente preenchidos e visíveis no verso do papel filtro. Por se tratar do mesmo procedimento de coleta para triagem neonatal, se uma amostra é coletada de forma inadequada ou se sua qualidade estiver prejudicada por procedimentos pós-coleta incorretos, a precisão dos resultados dos testes realizados fica comprometida. Nesses casos, as amostras são rejeitadas sem serem analisadas ${ }^{5,16}$.

Quanto a raça, nos resultados dos exames alterados $(\mathrm{n}=20), 50 \%$ das gestantes eram brancas, $40 \%$ pardas e $10 \%$ negras. No Brasil, a anemia falciforme é uma doença hematológica hereditária de alta prevalência entre negros e pardos, também ocorrendo entre brancos ${ }^{17}$. Estima-se que a prevalência de sujeitos com traço falciforme no Brasil é de $4 \%$, dentro da população geral, com nascimento anual estimado de 200 mil novos casos e com expectativa de haver, atualmente, 7.200.000 casos $^{18}$.

De acordo com os antecedentes gestacionais, 15\% apresentaram histórico de aborto pregresso à gestação atual. Esse fato pode ser relacionado com o resultado alterado do teste, pois existe a hipótese da existência de abortamento preferencial de embriōes com hemoglobina AA (normais) pelas mães com hemoglobina $S$ (AS). No entanto, essa hipótese só pode ser confirmada por técnicas de Biologia Molecular, além do excesso 
significativo de portadores do genótipo AA entre os embrióes abortados espontaneamente pelas heterozigotas com o traço falciforme ${ }^{17,19}$.

Assim, o seguimento dado a gestante diagnosticada com traços falciforme e hemoglobina $\mathrm{C}$ a partir do Teste da Mãezinha poderá ser de baixo risco. Entretanto, 5 gestantes foram referenciadas para alto risco podendo caracterizar a necessidade por alguma intercorrência, doença pré-existente ou adquirida durante a gestação. As mulheres portadoras de doença falciforme apresentam maior risco para morbidade materna e resultados perinatais adversos, quando comparadas às portadoras de traço falciforme. O cuidado adequado do binômio materno-fetal do início da gravidez até o puerpério é essencial para a assistência dessas gestaçóes ${ }^{13}$.

Observou-se que nas gestantes com traço falciforme e traço hemoglobina $\mathrm{C}$ analisadas, $80 \%$ apresentaram intercorrências, sendo mais frequentes a infecção do trato urinário $(50 \%)$, leucorreias em geral $(25 \%)$ e intercorrências durante no parto (12,5\%).

A infecção do trato urinário foi mais frequente em gestantes com traço falciforme. Nesse sentido, é necessário um olhar mais detalhado no pré-natal de gestantes com traço falcêmico ou hemoglobina $C^{6,9}$. As leucorreias mais comuns na gestação são: candidíase vulvovaginal, tricomoníase, clamídia e gonorreia. Casos diagnosticados devem ser tratados durante a gestação e reavaliados no puerpério para verificar a necessidade de novo tratamento. Em casos de recorrências frequentes, o(s) parceiro $(\mathrm{s}) \operatorname{deve}(\mathrm{m})$ ser avaliado $(\mathrm{s})$ quanto à necessidade de tratamento concomitante, exceto para tricomoníase, para a qual o parceiro sempre deve ser tratado ${ }^{12}$. Dessa forma, destaca-se que a triagem neonatal é um ponto fundamental na prevenção secundária da doença falciforme e demais complicaçôes que podem ocorrer durante a gestação $0^{20}$.

\section{Conclusão}

As hemoglobinopatias, quando diagnosticadas no inicio do pré-natal, são de grande relevância para uma assistência de qualidade, proporcionando uma gestação bem sucedida e um desenvolvimento fetal adequado. Nesse estudo observou-se baixa prevalência de hemoglobinopatias: $1,3 \%$ com o traço falciforme e $0,7 \%$ com traço de hemoglobina C. Entre as gestantes participantes, $80 \%$ apresentaram intercorrências no pré-natal, sendo as mais frequentes: infecção do trato urinário (50\%) e leucorreias em geral (25\%).

Diante dos resultados obtidos, o olhar do profissional de saúde para as gestantes diagnosticadas com traços falciformes e hemoglobina $\mathrm{C}$ deve se voltar para o planejamento das açôes do pré-natal. Nesse sentido, espera-se somar para a melhora da oferta do exame no Estado do Paraná, assim como elucidar possíveis questionamentos sobre a realização e a necessidade do exame na rede pública de saúde, para a identificação de possíveis gestantes de risco devido à hemoglobinopatias, visando minimizar intercorrências gestacionais e potencializando resultados positivos do pré-natal.

\section{Referências}

1. Carlos AM, Souza RAV, Souza BMB, Pereira GA, Tostes JS, Martins PRJ, et al. Hemoglobinopathies in newborns in the southern region of the Triângulo Mineiro, Brazil. Crosssectional study. Sao Paulo Med J. 2015;133(5):439-44.

2. Dos Santos PND, Freire MHS, Zanlorenzi GB, Pianovski MA, Denardi MAFV. Anemia falciforme: caracterização dos pacientes atendidos em um ambulatório de referência. Cogitare Enferm. 2014;19(4):755-93.

3. Tavares CFF, Guimarães JDS, Souza AM. Prevalence of hemoglobinopathies in school children: the importance of using confirmatory methods. Braz J Pharm Sci. 2015;51(2):361-6.

4. Viana-Baraciol LM, Bonini-Domingos CR, Pagliusi RA, Naoum PC. Prevenção de hemoglobinopatias a partir do estudo em gestantes. Rev Bras Hematol Hemoter. 2001;23(1):31-9.

5. Brasil. Ministério da Saúde. Secretaria de Atenção à Saúde. Departamento de Atenção Especializada. Manual de educação em saúde: Linha de cuidado em doença falciforme [Internet]. Brasília, DF: 2009 [citado em 2016 abr 10]. Disponível em: http://bvsms.saude.gov.br/bvs/publicacoes/ manual_educacao_saude_v2.pdf

6. Brasil. Ministério da Saúde. Secretaria de Atenção à Saúde. Departamento de Atenção Especializada. Coordenação da Política Nacional de Sangue e Homoderivados. Manual de Condutas Básicas na Doença Falciforme [Internet]. Brasília, DF: 2006 [citado em 2017 mar 3]. Disponível em: http:// www.riocomsaude.rj.gov.br/Publico/MostrarArquivo. aspx?C=Y0\%2BU19IsUXc\%3D

7. Paraná. Comissão Intergestores Bipartite do Paraná. Deliberaçáo no 330, de 28 de novembro de 2012. Aprova a realização do exame eletroforense de hemoglobina para os municípios que aderiram a Rede Mãe Paranaense. Curitiba, 2012.

8. Paraná. Secretaria de Estado de Saúde. Lei Estadual $n^{\circ}$ 867/1987. Torna obrigatório o Teste do Pezinho em todos os recém-nascidos do estado. Curitiba, 1987.

9. Alves AC, Da Silva FC, Oselame GB, Neves EB. Prevalência de pólipos colorretais em pacientes submetidos à colonoscopia em Curitiba, Brasil. Rev Bras Med. 2014;71(10).

10. Gil AC. Métodos e técnicas de pesquisa social. São Paulo: Atlas; 2010.

11. Brasil. Ministério da Saúde. Departamento de Açôes Programáticas Estratégicas. Secretaria de Atenção à Saúde. 
Pré-natal e puerpério: atenção qualificada e humanizada: manual técnico. Brasília, DF: Ministério da Saúde; 2005.

12. Brasil. Ministério da Saúde. Conselho Nacional de Saúde. Resolução no 466, de 12 de dezembro de 2012. Estabelece normas reguladoras para pesquisas e testes em seres humanos. Diário Oficial da União. Brasília, DF; 13 jun. 2013. Seção 1, p. 59.

13. Dos Santos ADP, Oselame GB, De Almeida DD, Da Silva TAA, De Oliveira EM. Caracterização de gestantes em atendimento pré-natal. Rev da Universidade Vale do Rio Verde. 2015;13(2):630-8.

14. De Galiza NGC, Da Silva PM. Aspectos moleculares da anemia falciforme. J Bras de Patologia e Med Lab. 2003;39(1):51-6.

15. Secretaria de Atenção à Saúde. Ministério da Saúde. Manual de normas técnicas e rotinas operacionais do Programa Nacional de Triagem Neonatal. Ministério da Saúde Brasília, DF: 2002.
16. Nomura RMY, Igai AMK, Tosta K, Fonseca GHHD, Gualandro SFM, Zugaib M. Resultados maternos e perinatais em gestaçôes complicadas por doenças falciformes. Rev Bras Ginecol Obstet. 2010;32(8):405-11.

17. Dos Santos TN, Barbosa MC, Dos Santos TEDJ, Damasceno D, Diniz S, Lemos VP. Triagem para hemoglobinas variantes em população adulta no Estado do Ceará. Revista Guará. 2015;1(3):131-9.

18. Nascimento MDLP. Abortos em mulheres portadoras de hemoglobina $S$ (AS). Rev Bras Hematol Hemoter. 2000;22(3):424-.

19. Ramalho AS, Silva ID, Pagotti M, Teixeira RC. Abortamentos espontâneos em portadoras do Traço Falciforme (AS). Rev bras hematol hemoter. 2003;25(4):265-6.

20. Silva CA, Baldim LB, Nhoncanse GC, Estevão IF, Melo DG. Neonatal screening program for hemoglobinopathies in the city of São Carlos, state of São Paulo, Brazil: analysis of a series of cases. Revista Paulista de Pediatria. 2015;33(1):19-27.

\section{Como citar este artigo:}

Liberato KMM, Oselame GB, Neves EB. Hemoglobinopatias em gestantes submetidas ao Teste da Mãezinha na rede pública de saúde. Rev. Aten. Saúde. 2017;15(51):46-51. 